Ophthalmologe 2020 $117: 307-312$ https://doi.org/10.1007/s00347-019-01029-w Online publiziert: 7. Januar 2020

c) Der/die Autor(en) 2020

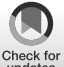

\author{
F. Bucher ${ }^{1} \cdot$ P. Mussinghoff ${ }^{2} \cdot$ T. Kühn $^{3} \cdot$ A. Stahl ${ }^{4} \cdot$ D. Böhringer ${ }^{1}$ \\ ${ }^{1}$ Klinik für Augenheilkunde, Universitätsklinikum Freiburg, Freiburg, Deutschland \\ ${ }^{2}$ Augenzentrum am St. Franziskus-Hospital Münster, Münster, Deutschland \\ ${ }^{3}$ ContraCare GmbH, Fürth, Nürnberg, Deutschland \\ ${ }^{4}$ Klinik und Poliklinik für Augenheilkunde, Universitätsmedizin Greifswald, Greifswald, Deutschland
}

\title{
Technische Umsetzung der Qualitätssicherung bei der intravitrealen operativen Medikamenteneingabe (IVOM)
}

Netzhautdicke im Verlauf operationalisieren. Zusätzliche Qualitätsindikatoren können über Frequenzanalysen, z. B. die Frequenz der IVOM im Verlauf oder die Anzahl durchgeführter OCT(optische Kohärenztomographie)-Kontrolluntersuchungen abgeleitet werden. Komplikations- und Abbruchraten spielen hier ebenfalls eine wichtige Rolle [8]. Für eine einheitliche Bewertung aller Qualitätsdimensionen ist die Zusammenführung und Standardisierung der Untersuchungs- und Behandlungsdaten angezeigt [6].

Die räumliche Trennung von Vor- und Nachuntersuchungen sowie IVOM stellt hohe Anforderungen an die netzwerkbasierte Integration der apparativen Ausstattung. Weiterhin ist aufgrund der hohen Arbeitsdichte bei den Leistungserbringern eine möglichst nahtlose Integration in den Arbeitsalltag mit benutzerfreundlicher Oberfläche wünschenswert.

Im Folgenden sollen die technischen und regulatorischen Anforderungen einer übergreifenden Plattform zur Ablage von strukturierten Daten inklusive der umfangreichen Bilddokumente aus der optischen Kohärenztomographie beleuchtet werden. Im Kontext der Informationssicherheit und einer revisionssicheren Dokumentation soll diese Arbeit orientierende Lösungsvorschläge unterbreiten.

\section{Technische Aspekte}

\section{Netzwerkstruktur}

Für die Datenfernübertragung bietet sich aufgrund der flächendeckenden Verfügbarkeit und der hohen Übertragungsgeschwindigkeiten die Infrastruktur des Internets an. Web-basierte Anwendungen bieten die idealen Voraussetzungen für eine abgesicherte zentrale Datenspeicherung in Kombination mit einer dezentralen Datenerfassung, da sowohl eine Verschlüsselung der Kommunikation als auch eine Verifikation der Kommunikationspartner gewährleistet werden können. Entsprechend sind Web-basierte Fallberichtsformulare inzwischen das bevorzugte Verfahren für die Datenerfassung in klinischen Studien [2]. Da die Web-basierten Anwendungen weltweit „sichtbar“ und damit potenziellen Angreifern gegenüber ständig exponiert sind, reicht eine Absicherung durch Passwörter alleine nicht aus. Zusätzliche Sicherheit schaffen sog. Zwei-FaktorAuthentifizierungsverfahren [10]. Hier tritt zu dem ersten Faktor „Passwort“ ein zweiter, hiervon unabhängiger Faktor. Es hat sich bewährt, dafür physikalische Objekte, beispielsweise ein Smartphone oder einen Token-basierten Schlüsselanhänger, zu wählen. Bei jedem Login auf die Web-Anwendung werden sowohl das Passwort als auch der Besitz des zweiten Faktors überprüft (beispielsweise Zustellung einer Zufalls-PIN an eine 
fest hinterlegte Mobilfunknummer, die dann zusätzlich zum Passwort abgefragt wird).

Für eine nahtlose Integration in den Arbeitsalltag sind rein Web-basierte Applikationen in der Regel nicht geeignet. Dies liegt daran, dass diese Programme aufgrund in den Browser eingearbeiteter Sicherheitskonzepte nicht direkt auf die Informationstechnologie vor Ort zugreifen können [11]. Insbesondere lassen sich die ophthalmologischen Geräte, wie beispielsweise die OCT-Bildgebungssysteme, nicht direkt aus dem Web heraus ansteuern.

Ein Lösungsansatz besteht in virtuellen privaten Netzwerken (VPN). Über ein VPN kann das lokale Computernetzwerk beliebig erweitert und der Datenverkehr verschlüsselt werden. Ein Beauftragter der Qualitätssicherung könnte auf diesem Weg beispielsweise direkt auf die Software des OCT-Bildgebungssystems zugreifen und dort die originalen Bildaufnahmen einsehen. Aufgrund der Implikationen des Datenschutzes und der Betriebssicherheit (s. Abschnitt „Regulatorische Aspekte bei der gemeinsamen Nutzung von Gesundheitsdaten“) ist dieser Ansatz ohne zusätzliche Sicherungsmaßnahmen nur für geschlossene Anwenderkreise, wie beispielsweise medizinische Versorgungszentren (MVZ), anwendbar.

\section{\) Über ein VPN kann das lokale Computernetzwerk erweitert und der Datenverkehr verschlüsselt werden}

Alternativ zu VPN-basierten Lösungen können Hardware- oder Softwareinstanzen in den beteiligten Einrichtungen implementiert werden, die einerseits per Schnittstellen (die internationalen Standards entsprechen, wie z. B. Gerätedatentransfer[GDT]- bzw. Health Level 7 [HL7]-Schnittstelle) Patientenstammdaten sowie klinische Daten aus den Primärsystemen der Kliniken und Praxen (Krankenhausinformationssysteme [KIS-Systeme] bzw. Praxisverwaltungssysteme [PVS-Systeme]) an die zentrale Qualitätssicherungsplattform übertra- gen und andererseits per Schnittstelle aus der Software des OCT-Gerätes die Bilddaten im jeweiligen proprietären Datenformat (z. B. e2e-files bei Heidelberg Engineering, Heidelberg, Deutschland, .fda-files bei Topcon, Tokio, Japan usw.) zuordnen. Zusätzlich zur Datenübertragung kann eine Pseudonymisierung der Daten erfolgen.

Der früher im Gesundheitswesen übliche Datenträgeraustausch (über CDROM oder USB-Datenträger) stellt trotz aller Vorzüge hinsichtlich der Datensicherheit aufgrund der fehlenden Interaktivität, des Zeitverzugs und des administrativen Mehraufwandes keine sinnvolle Alternative mehr dar.

\section{Erfassung von Behandlungsdaten}

Die Kontrolluntersuchungen nach IVOM erfolgen in Deutschland in der Regel nicht durch den Operateur, sondern durch konservativ tätige Augenärzte. Die medizinischen Daten werden daher an vielen unterschiedlichen Standorten erhoben. Insofern besteht die Herausforderung, dass der gleiche Sachverhalt zum Teil unterschiedlich dokumentiert wird. Dies ist besonders bei der Sehschärfe kritisch, da diese einen wichtigen Parameter für die Ergebnisqualität darstellt [3]. Einheitliche Kodiervorschriften können diesen Aspekt jedoch lösen, beispielsweise durch Umrechnung der Sehschärfenwerte in „logMAR“, die auch im Niedrigvisusbereich definiert ist, und eine verpflichtende standardisierte Dokumentation der Refraktionswerte, mit denen der Visus erhoben wurde. Weiterhin bieten sich im Interesse der besseren Vergleichbarkeit die Verwendung von Kodiersystemen wie Operationen- und Prozedurenschlüssel (OPS), „International Classification of Diseases“ (ICD), Alpha-ID (differenzierte Diagnosekodes) oder Human Phenotype Ontology (HPO) an. Überdies sollten die Eingabemasken die Dokumentation durch automatische Vorschläge oder die Darstellung möglicher Werteausprägungen unterstützen, beispielsweise durch Dropdown-Menüs.

Für eine automatisierte Übertragung der Nachbehandlungsdaten zur Qualitätssicherungsplattform „im Hin- tergrund“ bieten sich medizininformatische Standards wie „Fast Healthcare Interoperability Resources“ (FHIR) an [12].

\section{Erfassung von Bilddaten}

In der Qualitätssicherung bei IVOM nehmen die Aufnahmen aus der OCT eine Schlüsselrolle ein, da diese eine wichtige Grundlage für die Indikation der Injektionen darstellen. Es handelt sich dabei um umfangreiche Schnittbilddatensätze mit einer Dateigröße von bis zu $50 \mathrm{Me}$ gabytes pro Aufnahme. Einzelschnitte können zwar in $\operatorname{PDF}($ „Portable Document Format")-Formaten abgebildet und übermittelt werden. Dies führt jedoch im Vergleich zu Volumenscans zu einem deutlichen Informationsverlust und Qualitätsverlust, Aktuell befinden sich in Deutschland über 10 unterschiedliche retinologische OCT-Bildgebungssysteme auf dem Markt, sodass die Qualitätssicherung bei IVOM mit zahlreichen proprietären Dateiformaten konfrontiert ist. Konkret bedeutet dies, dass die qualitätssichernde Stelle - sofern die im Rahmen der Qualitätssicherung zu treffende Beurteilung auf Basis der „OCTVolumenscans" und nicht auf Basis von Ausdrucken im PDF erfolgen soll - die Softwarelösungen der jeweiligen OCTGerätehersteller vorhalten muss. Die dadurch bedingten Anforderungen an das Datenmanagement und nicht zuletzt die Kosten im Rahmen von Qualitätssicherungsmaßnahmen sind sehr hoch. Abhilfe kann hier eine Standardisierung von Datenformaten schaffen, die im Bereich der radiologischen Bildgebung längst umgesetzt ist.

》) Mithilfe eines DICOM-Viewers kann eine OCT-Untersuchung herstellerunabhängig ausgewertet werden

Vom „Digital Imaging and Communications in Medicine"-Komitee (DICOM Working Group 9 der „National Electrical Manufacturers Association“, NEMA) wurde 2007 ein Standard für OCT-Bilddateien definiert. Dieser beinhaltet die 
Möglichkeit, eine OCT-Untersuchung herstellerunabhängig unter Einsatz eines DICOM-Viewers auszuwerten. Der Standard enthält die Darstellung des Fundusbildes mit den einzelnen Schichtbildern („B-Scan“) mit Markierung der Position auf dem Fundusbild. Dieser Standard wurde im Jahr 2017 wesentlich erweitert, indem zusätzlich zu Fundusbild, B-Scan und Position im Fundus auch Segmentierungsinformationen ergänzt wurden [13]. Über diese Mitgabe von Segmentierungen sind auch vergleichende Berechnungen zwischen unterschiedlichen OCT-Herstellern realisierbar. Durch „Supplement 197“ hat die DICOM Working Group 9 zudem den Standard um die OCT-Angiographie erweitert. Neben dem erweiterten DICOM-OCT-Standard wurde jüngst in Bezug auf Messwerte in OCT-Untersuchungen im Rahmen einer Initiative der IHE (Integrating the Healthcare Enterprise) mit den „Key Measurements in ePDF“ ein Standard implementiert, der es erlaubt, diskrete Werte wie Netzhautdicken oder -volumenwerte strukturiert $\mathrm{zu}$ speichern und über Abfragen verfügbar $\mathrm{zu}$ machen. Bislang wurden diese Werte üblicherweise erst beim Betrachten berechnet. Der Key MeasurementStandard bietet jetzt die Möglichkeit, die Messwerte in einer Datenbank abzulegen und Verläufe direkt im Patienteninformationssystem darzustellen. Auch Abfragen durch qualitätssichernde Einrichtungen sind damit umsetzbar. Dieser Standard wird bereits von einzelnen Herstellern implementiert. Dennoch ist ein Vergleich von OCT-Daten unterschiedlicher Hersteller nicht direkt möglich und sollte von Herstellern wie auch regulierenden Organen mit Nachdruck verfolgt werden. Diese Bereitschaft zur Umsetzung internationaler Standards wird die Durchführung von Qualitätssicherungsmaßnahmen erheblich erleichtern. Die Herausforderung, dass in den DICOMOCT-Dateien in der Regel keine über Herstellergrenzen hinweg vergleichbare Analysen, wie beispielsweise die zentrale Netzhautdicke, hinterlegt sind, bleibt eine Anforderung an die OCT-Hersteller. Aktuell ist eine Therapieentscheidung auf Grundlage von OCT-Dateien unterschiedlicher Hersteller im Verlauf noch

Ophthalmologe 2020 · 117:307-312 https://doi.org/10.1007/s00347-019-01029-w

(c) Der/die Autor(en) 2020

F. Bucher · P. Mussinghoff · T. Kühn · A. Stahl · D. Böhringer

Technische Umsetzung der Qualitätssicherung bei der
intravitrealen operativen Medikamenteneingabe (IVOM)

Zusammenfassung

Hintergrund. Eine erfolgreiche Qualitätssicherung bei der intravitrealen operativen Medikamenteneingabe (IVOM) setzt eine komplexe informationstechnologische Infrastruktur voraus. Eine ortsungebundene Datenverfügbarkeit, die Standardisierung klinischer Daten sowie die Integration umfangreicher und aktuell nicht standardisierter Bilddokumente aus der Kohärenztomographie sowie die Einhaltung datenschutzrechtlicher Vorgaben stellen wesentliche Herausforderungen dar. Ziel der Arbeit. In diesem Beitrag werden technische und datenschutzrechtliche Grundsätze bei der technischen Implementierung beleuchtet.

Material und Methoden. Es erfolgt eine systematische Darstellung und Diskussion wesentlicher Teilaspekte bei der Implementierung einer Qualitätssicherung bei IVOM.

Ergebnisse. Im Bereich der Netzwerkstruktur haben Web-basierte Anwendungen ergänzt um lokale virtuelle private Netzwerke (VPN) und/oder andere Softwareinstanzen den früher üblichen Datenträgeraustausch ersetzt. Bei der Erfassung von Behandlungsdaten spielt die Standardisierung der Daten z. B. durch Umwandlung der Visusdaten in "logMAR" eine wichtige Rolle. Multiple optische Kohärenztomographen mit nicht standardisierten Datenformaten erschweren die Anbindung an eine allgemeine Qualitätssicherungsstruktur sowie die Vergleichbarkeit der Daten.

Diskussion. Internationale Standards werden dies in der näheren Zukunft vermutlich erleichtern. Bis dahin müssen individuelle Lösungen vor Ort gefunden werden.

\section{Schlüsselwörter}

Netzwerkstruktur · Behandlungsdaten . Datenschutz - Standardisierte Datenformate . Datenverfügbarkeit

\section{Technical aspects of quality assurance for intravitreal injections (IVI)}

Abstract

Background. Successful quality assurance in intravitreal injection (IVI) of medications requires a complex information technology infrastructure. The main challenges are data availability independent of location, standardization of clinical data, integration of extensive and currently non-standardized image documentation from coherence tomography and compliance with data protection regulations.

Objective. In this article the technical implementation and data protection principles are reviewed.

Material and methods. Essential aspects in the implementation of quality assurance in the field of IVI are discussed in a systematic approach.

Results. In the field of network architectures web-based applications supplemented by local virtual private networks (VPN) and/or other software instances have recently replaced the previously commonly used physical data medium exchange. The standardization of the data, e.g. by converting the visual acuity into logMAR, plays an important role in the collection of treatment data. Multiple non-standardized data formats in optical coherence tomography complicate the general quality assurance structure and comparability of data.

Conclusion. International standards will probably facilitate this in the near future. Until then individual solutions have to be found on site.

Keywords

Network architecture $\cdot$ Treatment data $\cdot$ Data protection $\cdot$ Standardized data formats $\cdot$ Data availability 
erheblich erschwert, insbesondere auch, da die Aufnahmeraster nicht standardisiert sind. Somit lassen sich identische Netzhautregionen nicht im Verlauf direkt vergleichen, wenn die Bilder mit unterschiedlichen Fabrikaten aufgenommen wurden.

Multiplattformbildbetrachter, die OCT-Volumenscans von unterschiedlichen Herstellern verarbeiten und anzeigen können, überwinden diese Einschränkungen teilweise, wurden bisher aber nur prototypisch implementiert [14]. Da es hierbei jedoch zu einer Verarbeitung der Originaldaten kommt, sind im klinischen Einsatz insbesondere das Medizinprodukterecht und die CE-Konformitätserklärung von medizinischen Systemen zu beachten. Die Umsetzung der oben genannten DICOM-Standards, die zu einer direkten Vergleichbarkeit der Originaldaten führen würde, kann hier einen praktikableren Lösungsweg darstellen. Aktuell ist es daher ratsam, die zulässigen OCT-Geräte für ein IVOMQualitätssicherungsprojekt explizit zu definieren.

\section{Regulatorische Aspekte bei der gemeinsamen Nutzung von Gesundheitsdaten}

Gesundheitsdaten werden gemäß Artikel 9 der Datenschutzgrundverordnung aus dem Jahr 2018 als kritische Kategorie eingestuft [9]. Für den Umgang mit gesundheitsbezogenen Daten im Rahmen der medizinischen Routineversorgung und Qualitätssicherung ist eine Rechtsgrundlage gegeben (Datenschutzgrundverordnung Artikel 9, Ziffern $2 \mathrm{~h}$ und 2i). Falls die Daten darüber hinaus verarbeitet oder übertragen werden, wie z. B. an eine qualitätssichernde Einrichtung, die mehrere medizinische Versorgungseinheiten betreut, ist eine explizite Einwilligung erforderlich. Abhängig von den zugrunde liegenden Abrechnungsregeln (EBM [Einheitlicher Bewertungsmaßstab], Selektivvertrag) sind die jeweiligen kollektiv- bzw. selektivvertraglichen Bestimmungen $\mathrm{zu}$ beachten. Grundsätzlich muss für alle Datenverarbeitungsprozesse ein Datenschutzkonzept erstellt und mit dem zuständigen Datenschutzbeauftragten abgestimmt werden.

Im Kontext der Informationssicherheit ist für die Qualitätssicherung zusätzlich entscheidend, dass die zu prüfenden Daten revisionssicher gespeichert sind. Es muss jederzeit nachvollziehbar sein, wer wann, was geändert bzw. gelöscht hat. Für den Fall, dass dies vor Ort nicht auf technischem Weg umsetzbar ist, empfiehlt sich eine vertragliche Verpflichtung zur manuellen Dokumentation aller Änderungen.

Falls Ärzte mit personenbezogenen Daten forschen wollen, muss darüber hinaus gemäß der Berufsordnung bei der zuständigen Ethikkommission zwingend ein Ethikvotum eingeholt werden oder eine Anonymisierung im Vorfeld erfolgen [15].

\section{Diskussion}

In diesem Beitrag wurden technische und regulatorische Aspekte der Qualitätssicherung bei IVOM schlaglichtartig beleuchtet.

Die Qualitätssicherung weist in der Regel für den einzelnen Behandlungsfall keinen direkten Nutzwert auf. Daher wird diese von den Leistungserbringern, die gerade bei der IVOM aufgrund der hohen Fallzahlen unter hohem Zeitdruck arbeiten müssen, nicht selten als lästige Pflicht empfunden. Mit der Qualitätssicherung verbundene Chancen einer gesamtheitlichen Prozessoptimierung, die die Handlungsabläufe nicht nur beschleunigen, sondern auch die Fehlerminimierung für künftige Behandlungsfälle ermöglichen, bleiben oftmals ungenutzt. Um angemessene Qualitätssicherung zu gewährleisten und Optimierungspotenziale zu bergen, sollte eine Integration in die vor Ort bestehenden informationstechnologischen Strukturen erfolgen.

\section{》) Mit der Qualitätssicherung verbundene Chancen einer Prozessoptimierung bleiben oftmals ungenutzt}

Wesentliche Herausforderungen finden sich dabei aktuell in der nicht standar- disierten Vielfalt der $\mathrm{zu}$ integrierenden Systeme (bezüglich Visus- und OCTGeräten) sowie den variierenden Prozessanforderungen im Rahmen individueller Strukturverträge. Gerade Praxisund Versorgungsnetze mit mehreren unabhängigen Standorten und ggf. unterschiedlicher technischer Ausstattung stehen vor der Herausforderung, unterschiedliche Datenformate in einer Dateninfrastruktur zu integrieren. Aus Sicht der Versorgungsnetze sind die initiale Bereitstellung, v. a. aber auch die Pflege einer solchen Infrastruktur kostenintensiv (bei Verwendung kommerziell erwerblicher Plattformen) oder zeitintensiv. Gleichzeitig werden Einzelstandorte mit dem Streben nach Anschluss an ein Versorgungsnetzwerk auf Anschaffung bestimmter integrationsfähiger Systeme eingeschränkt und zur Pflege der Schnittstelle verpflichtet. Mit Blick auf die Strukturverträge muss verhindert werden, dass die Leistungserbringer mit einer Vielzahl von technischen Lösungen konfrontiert werden, im Extremfall eine pro Strukturvertrag. Dies würde neben dem Lernaufwand und erhöhter Fehleranfälligkeit auch einen hohen technischen Installations- und Wartungsaufwand vor Ort bedingen. Bis heute ist in Deutschland keine sektorenübergreifende Plattform allgemein verfügbar. International hat sich das „Save Sight Registry“ als vielversprechender Ansatz etabliert, der sich als Vorlage für zukünftige Entwicklungen in Deutschland anbietet [5]. Auch die Deutsche Ophthalmologische Gesellschaft baut mit „oregis“ eine überregionale technische Struktur auf, die sich als Plattform für eine allgemeine Qualitätssicherung bei IVOM eignen könnte [16].

Bis dies so weit ist, können die in diesem Heft besprochenen „Leuchtturmprojekte“ als Anregungen für eigene Lösungen dienen.

\section{Fazit für die Praxis}

- Web-basierte Anwendungen, ergänzt um virtuelle private Netzwerke (VPN) oder andere lokale Softwarelösungen, erfüllen die Anforderungen ortsungebundener Datenverfügbarkeit an Netzwerksysteme und stellen 
damit State-of the-Art-Lösungen für eine funktionierende Qualitätssicherung dar.

- Eine standardisierte Dokumentation von Behandlungsdaten, wie z. B. Visuswerte in "logMAR", ist für die Vergleichbarkeit der Daten eine wichtige Voraussetzung.

- Ein direkter Vergleich von Bilddatensätzen optischer Kohärenztomographen (OCT) unterschiedlicher Hersteller ist aufgrund proprietärer Datenformate aktuell nicht möglich. Ein Vergleich von Einzelschnitten im „Portable Document Format" (PDF) ist möglich, geht aber mit einem wesentlichen Informations- und Qualitätsverlust einher. Eine Definition zulässiger OCT-Geräte für ein Qualitätssicherungsprojekt stellt eine Lösung dar.

- Für die Datenverarbeitungsprozesse im Rahmen der Qualitätssicherung muss zur Erfüllung der Datenschutzgrundverordnung ein Datenschutzkonzept erstellt werden.

\section{Korrespondenzadresse}

\section{Prof. Dr. D. Böhringer}

Klinik für Augenheilkunde, Universitätsklinikum Freiburg

Killianstr. 5, 79106 Freiburg, Deutschland Daniel.boehringer@uniklinik-freiburg.de

\section{Einhaltung ethischer Richtlinien}

Interessenkonflikt. F. Bucher, P. Mussinghoff, T. Kühn, A. Stahl und D. Böhringer geben an, dass kein Interessenkonflikt besteht.

Für diesen Beitrag wurden von den Autoren keine Studien an Menschen oder Tieren durchgeführt. Für die aufgeführten Studien gelten die jeweils dort angegebenen ethischen Richtlinien.

Open Access Dieser Artikel wird unter der Creative Commons Namensnennung 4.0 International Lizenz veröffentlicht, welche die Nutzung, Vervielfältigung, Bearbeitung, Verbreitung und Wiedergabe in jeglichem Medium und Format erlaubt, sofern Sie den/die ursprünglichen Autor(en) und die Quelle ordnungsgemäß nennen, einen Link zur Creative Commons Lizenz beifügen und angeben, ob Änderungen vorgenommen wurden.

Die in diesem Artikel enthaltenen Bilder und sonstiges Drittmaterial unterliegen ebenfalls der genannten Creative Commons Lizenz, sofern sich aus der Abbildungslegende nichts anderes ergibt. Sofern das betreffende Material nicht unter der genannten Creative Commons Lizenz steht und die betreffende Handlung 
nicht nach gesetzlichen Vorschriften erlaubt ist, ist für die oben aufgeführten Weiterverwendungen des Materials die Einwilligung des jeweiligen Rechteinhabers einzuholen.

Weitere Details zur Lizenz entnehmen Sie bitte der Lizenzinformation auf http://creativecommons.org/ licenses/by/4.0/deed.de.

\section{Literatur}

1. HolzFG, Tadayoni R, Beatty Setal (2016) Key drivers of visual acuity gains in neovascular age-related macular degeneration in real life: findings from the AURA study. Br J Ophthalmol 100:1623-1628

2. Le Jeannic A, Quelen C, Alberti C et al (2014) Comparison of two data collection processes in clinical studies: electronic and paper case report forms. BMC Med Res Methodol 14:7

3. Mbagwu M, French DD, Gill M et al (2016) Creation of an accurate algorithm to detect Snellen best documented visual acuity from ophthalmology electronic health record notes. JMIR Med Inform 4:e14

4. Mehta H, Tufail A, Daien V et al (2018) Real-world outcomes in patients with neovascular age-related macular degeneration treated with intravitreal vascular endothelial growth factor inhibitors. Prog Retin Eye Res 65:127-146

5. Nguyen V, Leung KFC, Nguyen CL et al (2019) Assesing the accuracy of a large observational registry of neovascular age-related macular degeneration. Retina. https://doi.org/10.1097/ IAE.0000000000002485

6. Tan JCK, Ferdi AC, Gillies MC, Watson SL (2019) Clinical registries in ophthalmology. Ophthalmology 126:655-662

7. Wecker T, Grundel B, Reichl S et al (2019) AntiVEGF injection frequency correlates with visual acuity outcomes in pro re nata neovascular AMD treatment. Sci Rep 9:3301

8. Wintergerst MWM, Bouws J, Loss J et al (2018) Reasons for delayed and discontinued therapy in agerelated macular degeneration. Ophthalmologe 115:1035-1041

9. (2018) Reform der EU-Datenschutzvorschriften 2018. https://ec.europa.eu/commission/ priorities/justice-and-fundamental-rights/dataprotection/2018-reform-eu-data-protectionrules_de.Zugegriffen:25.Sept. 2019

10. BSI für Bürger - Zwei-Faktor-Authentisierung. https://www.bsi-fuer-buerger.de/ BSIFB/DE/DigitaleGesellschaft/OnlineBanking/ Zwei_Faktor_Authentisierung/Zwei-FaktorAuthentisierung_node.html. Zugegriffen: 25 . Sept. 2019

11. BSI für Bürger - Machen Sie Ihren Browser sicher. https://www.bsi-fuer-buerger.de/ BSIFB/DE/Empfehlungen/EinrichtungSoftware/ EinrichtungBrowser/Sicherheitsmassnahmen/ SicherheitsCheck/sicherheitscheck_node.html. Zugegriffen:25. Sept. 2019

12. Index - FHIR v4.0.0. https://www.hl7.org/fhir/. Zugegriffen:25. Sept. 2019

13. C.8.17.14 Ophthalmic Optical Coherence Tomography En Face Image Module. http://dicom. nema.org/medical/dicom/current/output/chtml/ part03/sect_C.8.17.14.html.Zugegriffen:25. Sept 2019

14. Bitbucket. https://bitbucket.org/uocte/uocte. Zugegriffen:25. Sept. 2019

15. (2018) (Muster-)Berufsordnung für die in Deutschland tätigen Ärztinnen und Ärzte. https://www.bundesaerztekammer.de/recht/ berufsrecht/muster-berufsordnung-aerzte/ muster-berufsordnung/. Zugegriffen: 25. Sept. 2019

16. oregis - Deutsches Ophthalmologisches Register. http://oregis.de/.Zugegriffen:25. Sept. 2019

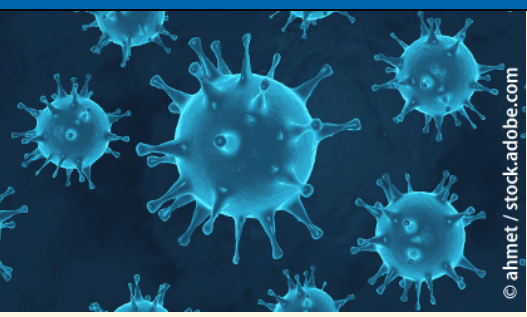

\section{Aktuelle Entwicklung zu \\ COVID-19 bei Springer Nature und Springer Medizin}

Springer Nature und Springer Medizin unterstützen die globale Reaktion auf die COVID-19-Pandemie, in dem ein schneller und direkter $\mathrm{Zu}$ gang zu den neuesten verfügbaren Forschungsergebnissen und Daten ermöglicht wird.

Auf der Homepage SpringerMedizin.de finden Sie ein immer aktuelles Dossier mit Beiträgen, Forschungsarbeiten und Ergebnissen zu SARS-CoV-2 sowie relevanten Links.

Darin z.B. auch die kürzlich publizierte

Empfehlung von DIVI, DGIIN, DGAI und DGP zur Intensivtherapie von Patienten mit COVID-19.

Springer Nature arbeitet mit globalen Organisationen zusammen, und verlinkt über SpringerNature.com/de auf eine eigene Landingpage mit einer Vielzahl an Information sowie freiem Zugriff auf die COVID-19-Contentplattformen von Nature Research, BioMed Central (BMC) und Springer.

Das Dossier zu Coronavirus / Covid-19 von Springer Medizin finden Sie hier: www.springermedizin.de/covid-19

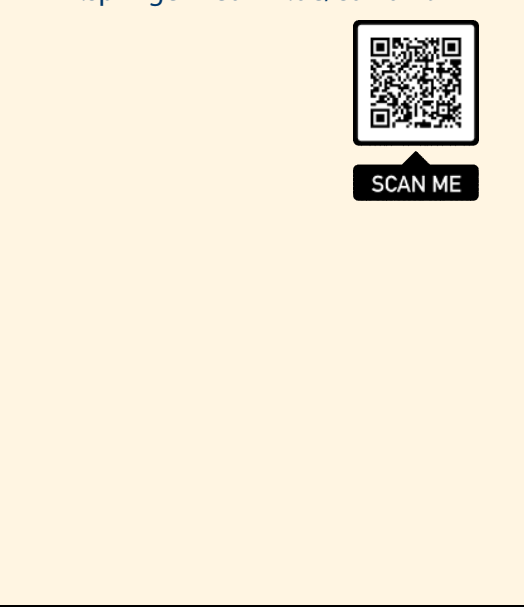

